\title{
BMJ Open What are the links between evidence- based medicine and shared decision- making in training programs for junior doctors? A scoping review protocol
}

\author{
Mary Simons (D) ," Frances Rapport (D) , ${ }^{2}$ Yvonne Zurynski (D) , Jeremy Cullis, ${ }^{1}$ \\ Andrew Davidson ${ }^{1}$
}

To cite: Simons M, Rapport F, Zurynski Y, et al. What are the links between evidence-based medicine and shared decisionmaking in training programs for junior doctors? A scoping review protocol. BMJ Open 2020;10:e037225. doi:10.1136/ bmjopen-2020-037225

- Prepublication history for this paper is available online. To view these files, please visit the journal online (http://dx.doi org/10.1136/bmjopen-2020037225).

Received 24 January 2020 Revised 25 February 2020 Accepted 07 April 2020

Check for updates

(c) Author(s) (or their employer(s)) 2020. Re-use permitted under CC BY-NC. No commercial re-use. See rights and permissions. Published by BMJ.

${ }^{1}$ Department of Clinical Medicine, Macquarie University, North Ryde, NSW 2019, Australia ${ }^{2}$ Health Implementation Science, Centre for Healthcare Resilience and Implementation Science, Australian Institute of Health Innovation, Macquarie University, North Ryde, NSW 2019, Australia

${ }^{3}$ Health System Sustainability, Australian Institute of Health Innovation and the NHMRC Partnership Centre for Health System Sustainability, Macquarie University, North Ryde, NSW 2019, Australia

Correspondence to

Ms Mary Simons;

mary.simons@mq.edu.au

\section{ABSTRACT}

Introduction Patient-centred care is pivotal to clinical practice and medical education. The practice of evidencebased medicine (EBM) and shared decision-making (SDM) are complementary aspects of patient-centred care, but they are frequently taught and reported as independent entities. To effectively perform all steps of EBM, clinicians need to include patients in SDM conversations, however, the uptake of this has been slow and inconsistent. A solution may be the incorporation of SDM into EBM training programmes, but such programmes do not routinely include SDM skills development. This scoping review will survey the literature on the kinds of EBM and SDM educational programmes that exist for recently qualified doctors, programmes that incorporate the teaching of both EBM and SDM skills, as well as identifying research gaps in the literature.

Methods and analysis Literature searches will be conducted in the databases Medline, Embase, Scopus and Cochrane Library. Bibliographies of key articles and their citing references will also be hand-searched and assessed for inclusion. Selected grey literature will be included. Papers must be written in English, or provide English abstracts, and date from 1996 to the present day. Two independent reviewers will screen titles and abstracts, check full texts of selected papers for eligibility and extract the data. Any disagreement will be resolved, and consensus reached, if necessary, with the assistance of a third reviewer. Qualitative and quantitative studies that address educational interventions for either EBM, SDM or both will be included. Data extraction tables will present bibliographic information, populations, interventions, context and outcomes. Data will be summarised using tables and figures and a description of findings. Ethics and dissemination This review will synthesise information from publicly available publications and does not require ethics approval. The results will be disseminated via conference presentations and publications in medical journals.

\section{INTRODUCTION}

The practice of evidence-based medicine (EBM) requires the clinician to use their clinical experience and expertise, along with the best current research evidence and
Strengths and limitations of this study

- Evidence-based medicine (EBM) and shared decision-making (SDM) are not routinely taught together to medical graduates making this a novel area of research to be addressed by a scoping review.

- The standards of educational programmes for EBM and SDM are inconsistent and poorly reported, yet both are core skill areas in providing and improving patient-centred care.

- This review will identify research gaps by assessing existing educational programmes where EBM and SDM skills are taught separately and together.

- Qualitative research methods may add uniquely informative and rich data to this area of inquiry, particularly where quantitative methods have not been fully used.

- SDM, as part of the EBM 'apply' process, may indicate the degree to which patient outcomes or experiences can be assessed following EBM training.

patient preferences when making healthcare decisions. ${ }^{1}$ This practice may also be termed evidence-based practice (EBP) or evidencebased healthcare (EBHC). EBM involves the clinician considering their own clinical expertise when using the best research evidence to decide on a patient care pathway. However, neither clinical experience or research evidence is enough on its own; for example, the use of a high level of evidence such as a randomised controlled trial is insufficient to determine a clinical decision; individual patient circumstances and preferences must be factored into the decision-making process, ${ }^{1}$ so that EBM becomes a personalised experience with the individual patient's needs at its core. Shared decision-making (SDM) takes this process further by proactively involving the patient in the clinical decision-making process. SDM is a process by which the patient and clinician collaborate to make healthcare 
decisions that consider the medical evidence, the clinician's expertise and the patient's values, preferences and circumstances. ${ }^{2}$ SDM encourages the patient to ask questions about their care options, while patient decision aids (tools that help people become involved in healthcare decision-making by providing information about the options and outcomes, and by clarifying personal values) can be used to clarify the risks and benefits of the available options. ${ }^{3}$

The role of EBM and SDM in improving patientcentred care has been increasingly recognised in recent years, but within separate domains. ${ }^{4}$ As such, EBM and SDM are seldom taught together even though they share core patient-centred principles such as the patient's individual preferences for care, their personal circumstances and the importance of engendering patient empowerment and autonomy during the care process.

\section{The challenges of EBM training}

Medical training and professional accreditation bodies acknowledge the role of EBM in supporting graduate capabilities to ensure a well-rounded doctor, who fulfils all three roles together: 'scientist', 'scholar' and 'healthcare practitioner'. ${ }^{5}$ Yet EBM training programmes have been criticised for their inconsistent content, poor reporting of interventions and inadequate evaluation of outcomes. Albarqouni and colleagues conducted a systematic review of the completeness of reporting of EBM training programmes and concluded that the overall standard of reporting was poor; none of the 83 studies identified included all aspects of their training programmes in the descriptions they offered (in particular, teaching materials, training providers, frequency of programmes being offered, timing and duration of training). ${ }^{6}$ The review concluded that without this information, translation into practice would be difficult. ${ }^{6}$ Another systematic review investigated what is taught and evaluated in controlled studies of EBM training, and found that most programmes included only some of the five EBM steps of: ask, acquire, appraise, apply and assess; with an emphasis on critical appraisal of the evidence (step three).$^{7}$ Only $10(12 \%)$ of the included 85 studies taught all five steps of EBM, skills evaluation across programmes was inconsistent and poor quality outcome measures were used. ${ }^{7}$ In addition, none of the studies attempted to measure any benefits to patients following EBM training. ${ }^{7}$ These findings are supported by an earlier expert commentary, which cited the difficulties of educational research in general, and the poor quality of evidence supporting the implementation of EBM training programmes. ${ }^{8}$ Contributing factors included: reliance on quantitative evaluation methods that cannot capture the complexity of training programmes, constantly changing student population samples, brief time-allocation for EBM teaching, and the many EBM programmes tailored to the needs of specific institutions which, as a result, were not readily transferable. ${ }^{8}$ A systematic review of instruments for measuring EBM learning outcomes found that most assessments were focused on the acquisition and appraisal of EBM steps, and many instruments lacked rigorous validity testing. ${ }^{9}$ Another systematic review found that the evaluation of EBM attitudes and behaviours lagged behind the evaluation of knowledge and skills; in particular, that there was a need to evaluate the way in which trainees apply the evidence to individual patients and incorporate patient preferences and circumstances. ${ }^{9}$ While EBM courses for doctors demonstrate short-term improvements in knowledge, there is no strong evidence linking EBM training to long-term knowledge improvement, changes in clinical practice or patient outcomes. ${ }^{10}$ With the growing recognition of the patient's role in clinical care, there is a need to incorporate meaningful patient outcome measures into the assessment of EBM learning and practice.

\section{Shared decision-making}

SDM is a means by which patient preferences and circumstances are incorporated into the decision-making process after clinician and patient discuss the options, benefits and risks of a healthcare decision. ${ }^{4}$ In so doing, SDM supports the practice of EBM. ${ }^{11}$ SDM is most appropriate when there are uncertainties as to the best option for treatment, tests or surveillance for the patient, or if there is more than one appropriate option. ${ }^{2}$ The essential steps of SDM outlined by Legare and Witteman are: first, the patient and clinician realise a decision needs to be made; second, there must be an understanding by both of the evidence in support of, or against, a particular decision and third, the decision taken must support the clinician's viewpoint and the patient's circumstances and preferences. ${ }^{2}$ The use of decision aids can facilitate the process of SDM by providing visual representations of the evidence, options available and risk factors. A Cochrane review reported the benefits for patients of using decision aids, including increased knowledge, autonomy and participation in their care. ${ }^{12}$ In addition, decision aids used as part of the SDM process reduced the proportion of indecisive participants and appeared to have a positive effect on patient-clinician communication. ${ }^{12}$ The Cochrane review also found that the practice of SDM using decision aids led to more conservative patient decisions, particularly where invasive elective surgery was an option. $^{12}$

\section{SDM: uptake and training programmes}

Although the practice of SDM is recommended by many key healthcare groups, its widespread uptake by clinicians has been slow. ${ }^{21314}$ This has been attributed to a range of issues including lack of training opportunities, ${ }^{13}$ time constraints, perceptions that SDM cannot be applied because of a patient's uniquely individual character and the nature of the clinical situation. ${ }^{2}$ The widespread belief that SDM takes up more time in a consultation has been refuted in a Cochrane systematic review that found only an extra $2.6 \mathrm{~min}$ is added to a standard consultation when SDM with decision aids is applied. ${ }^{12} \mathrm{~A}$ recent scoping review found that reports of interventions used 
to increase the uptake of SDM by healthcare professionals were inconsistent as they used multiple educational strategies, lacked learning needs-assessment and did not use standard outcome assessments. ${ }^{15}$ They also noted that SDM training is not routinely integrated into basic medical training or postgraduate professional development opportunities. ${ }^{15}$ Diouf and colleagues conducted an environmental assessment of the growth of training programmes in SDM and identified 94 new programmes created between 2011 and 2015, an increase of $174 \% .^{16}$ However, despite the growing awareness of SDM in clinical environments, the actual uptake by clinicians is low and training programmes are not rigorously evaluated; less than $25 \%$ of SDM training programmes between 1996 and 2015 have been evaluated, leading to difficulties establishing which training techniques are most effective. $^{16}$

Despite the apparent complementary nature of EBM and SDM in focusing on patient preferences to improve patient care, each skill set of EBM and SDM, recognised as an essential aspect of optimal clinical practice, and needed by the clinician to support patient-centred care, has evolved independently of the other, in a somewhat haphazard manner. '(EBM) and shared decision making (SDM) are both essential to quality health care, yet the interdependence between these 2 approaches is not generally appreciated' (4p1295).

\section{RATIONALE FOR REVIEW}

The parallel development, and yet lack of interdependency of EBM and SDM, has left gaps in the completeness of EBM practice, such as failure to take patient preferences into account using SDM, a need for more consistent training, and uptake and evaluation of SDM by institutions and clinicians. ${ }^{4}$ EBM is grounded in epidemiology, where the initial steps of asking a question, appraising and applying evidence are emphasised, but where little attention is given to discussions of the evidence with the patient. ${ }^{4}$ Exploring patient preferences via SDM is not usually taught or assessed as part of EBM training, and when SDM is taught, it is often presented within a separate communications programme. ${ }^{4}$

In summary, EBM practice has been criticised for relying too much on the best evidence, while minimising the role of patient preferences. Therefore, doctors need to practice the 'apply' step of EBM by using SDM with patients to incorporate their values and preferences, alongside the current evidence and the doctor's own experience, into patient care decisions.

This scoping review will seek to explore the degree to which training programmes and evaluation instruments address all EBM steps ${ }^{17}$ and include SDM to improve EBM programmes and practice. While patient outcomes can be difficult to attribute to EBM training and practice alone, evaluation of patient experience and SDM may provide a link between EBM training and patient outcomes.
The gaps in EBM practice and teaching are emerging at a time when patient empowerment is increasing, and patients are now seen as equal partners in their own healthcare. Thus, it is an opportune time for both movements (EBM and SDM) to collaborate and evolve together.

\section{OBJECTIVES FOR REVIEW}

This scoping review will investigate the ways in which EBM and SDM are taught to junior doctors and the degree to which their teaching takes place within the same training programme. A scoping review was chosen as the most appropriate format to address this question, as published reports of EBM educational programmes appear to overlook the joint teaching of SDM and EBM, thus indicating the need for the broad, inclusive search strategies afforded by a scoping review. Research gaps in the literature, such as ways of incorporating and measuring SDM skills as part of EBM training, may also be identified. The authors plan to identify priorities to improve clinicians' educational development and make recommendations to enhance future training of SDM and EBM.

\section{METHODS AND ANALYSIS}

The scoping review methods are guided by the framework created by Arksey and O'Malley ${ }^{18}$ which has widespread use among scoping review protocols, and has been extended by Levac and colleagues. ${ }^{19}$ According to this framework ${ }^{18}$ there are five methodological stages to follow:

Stage 1: identify the research question.

Stage 2: identify relevant studies.

Stage 3: select studies.

Stage 4: chart the data.

Stage 5: collate, summarise and report results.

The Preferred Reporting Items for Systematic Reviews and Meta-Analyses checklist for scoping reviews (PRIS$\mathrm{MA}-\mathrm{ScR})^{20}$ has guided the development of this protocol, by enabling scoping review methodology to be clearly documented. In addition specific sections of the Joanna Briggs Institute Reviewers' Manual (chapter 11.2) that cover the steps of the scoping review protocol include valuable elements for consideration for our study. ${ }^{21}$ For example, the section on inclusion criteria (11.2.4) describes detailed criteria relevant to our review including study type and presentation of results (11.2.8). ${ }^{21}$

\section{Research question}

The following primary research question will be investigated:

What are the links between EBM and SDM in training programmes for junior doctors?

\section{Secondary questions}

- Which EBM skills are taught to junior doctors?

- Which SDM skills are taught to junior doctors? 
- By what methods and modes are EBM and SDM taught to junior doctors?

- To what extent does the peer reviewed literature report on the inclusion and effectiveness of SDM in EBM educational programmes?

- To what extent can SDM outcomes be an indicator of patient experience or outcomes following EBM training that incorporates SDM?

The primary and secondary questions can be broken up into the following searchable elements: 'Population, Concepts, Context', according to the PRISMA-ScR. ${ }^{20}$ The population is junior doctors; defined as recent medical graduates who are undertaking further training as interns (most junior), residents, registrars or fellows (most senior). This terminology is based on the Australian medical education system and is further explained in the Author note. Junior doctors can be practising in any discipline within hospitals or community settings. The concepts are how EBM is taught to junior doctors, how SDM is taught to junior doctors and whether both EBM and SDM are taught together. The context consists of educational or professional development programmes for junior doctors. The outcomes include reported outcomes from educational programmes in EBM and SDM, such as EBM knowledge scores, EBM attitude survey results, patient experience data and patient reported outcomes data.

\section{Identification of relevant studies}

The following inclusion criteria must be met for studies to form part of this scoping review:

- Junior doctors must be either interns, residents, registrars or fellows working in any medical or surgical discipline (see Author note). Eligible junior doctors may work in a city or rural setting, or in a hospital or community environment.

- Studies must include educational instruction of either EBM, SDM or both.

- Programmes can be delivered in a didactic way or they can be integrated into the clinical environment.

- Qualitative and quantitative studies will be included.

- Studies must be written in English or provide English abstracts and must date from 1996 (when the term 'evidence-based medicine' first received widespread attention in the literature) to the present day.

Exclusion criteria include:

- Non-medical healthcare professional EBM educational programmes.

- Students (medical and non-medical), senior medical and surgical consultants.

- Studies that do not include an educational intervention.

- Studies written in languages other than English.

- Studies published before 1996.

\section{Information sources}

The following bibliographic databases, with available years indicated in brackets, will be searched using subject
Box 1 Search strategy. Ovid Medline

1. (Decision Making/ or patient participation/ or patient preference/ or Physician-Patient Relations/ or (patient preference* or patient participation or patient involvement or patient engagement or patient-clinician communication or patient-centred care).ti,ab.) and ((shared adj decision*) or SDM).ti,ab.

2. exp Evidence-based practice/ or (evidence-based practice* or evidence-based surgery or evidence-based medicine or evidencebased healthcare).ti,ab.

3. exp Specialties, surgical/ and (resident* or registrar* or fellow* or intern* or interns or internist).ti,ab.

4. (exp Medicine/ or faculty, medical/ or exp medical staff/ or medical staff, hospital/ or hospitalists/ or exp physicians/) and (resident* or registrar* or fellow* or intern or interns or internist).ti,ab.

5. Education, Medical, Continuing/ or education, medical, graduate/ or "internship and residency"/ or teaching rounds/ or curriculum/ or Teaching/ or Learning/ or (teach* or learn* or educat* or train* or curricul $^{\star}$ or development or course* or program*).ti,ab.

6. $\mathrm{or} / 1-2$

7. or $/ 3-4$

8. and $/ 5-7$

9. and $/ 1,5,7$

10. and $/ 2,5,7$

11. 0 r $/ 9-10$

12. limit 11 to (English language and $y r=" 1996-2020 ")$

headings and keywords for the following concepts: junior doctors, EBM, SDM, educational interventions: Ovid Medline (1946-2020), Embase (1974-2020), Scopus (1996-2020) and Cochrane Library (1999-2020). Scopus will be searched in addition to Embase due to the broader subject scope of this database, its keyword searching functionality and its currency. The bibliographies of key articles and their citing references will also be hand-searched in Scopus and assessed for inclusion if they meet the inclusion criteria.

To identify relevant documents in the grey literature, we will search a variety of sources including Open Grey: http://www.opengrey.eu/, the UK's National Health Service (NHS) website: https://www.england. nhs.uk/shared-decision-making/, Australian Commission on Safety and Quality in Healthcare: https://www. safetyandquality.gov.au/, the Canadian Foundation for Health Improvement https://www.cfhi-fcass.ca/ and other similar websites. Other possible sources include, but will not be limited to, the following: policy, guidelines or recommendation documents from government websites, doctors' associations, training organisations, health consumer organisations, peak bodies and conference abstracts.

\section{Search strategies}

A draft search strategy in Ovid Medline (1946-2020), provided in box 1 , will be included and adapted for searching other databases.

\section{Data management}

Results of database and handsearching will be exported to EndNote V.X9 (Clarivate Analytics, Pennsylvania, USA) 


\begin{tabular}{|c|c|}
\hline Data item & Description \\
\hline Authors & Study authors \\
\hline Title/year & Article details \\
\hline Country & Geographical location \\
\hline Population & Doctors in training (see Author note) \\
\hline Size & Sample size \\
\hline Design & $\begin{array}{l}\text { Study design: qualitative, quantitative, mixed, } \\
\text { cohort, prospective, retrospective, observational }\end{array}$ \\
\hline Setting & $\begin{array}{l}\text { Educational setting: classroom, ward, } \\
\text { workshop, programme, course, module, lecture, } \\
\text { online }\end{array}$ \\
\hline Content & $\begin{array}{l}\text { Educational content: EBM, EBP, EBHC, } \\
\text { evidence-based surgery, SDM, }\end{array}$ \\
\hline Delivery & $\begin{array}{l}\text { Creation, delivery and analysis of educational } \\
\text { interventions }\end{array}$ \\
\hline Outcomes & $\begin{array}{l}\text { Classified according to Barr et al. }{ }^{23} \text { Level } 1 \text { : } \\
\text { learners' reaction. Level 2a: modification of } \\
\text { attitudes/perceptions. Level } 2 \text { b: acquisition of } \\
\text { knowledge/skills. Level 3: change in behaviour. } \\
\text { Level 4a: change in organisational practice. } \\
\text { Level 4b: benefits to patients/clients. }\end{array}$ \\
\hline
\end{tabular}

EBHC, evidence-based healthcare; EBM, evidence-based medicine; EBP, evidence-based practice; SDM, shared decisionmaking.

and results for final analysis will be exported to an Excel Spreadsheet.

\section{STUDY SELECTION}

Two researchers will independently screen the results according to eligibility criteria at each stage of the process (title, abstract, full text) to create a transparent means of study selection. A third researcher will arbitrate in the event of any disagreement between reviewers to ensure a consensus can be reached. A PRISMA flow diagram will be included to depict the study selection process at this stage. $^{22}$

\section{DATA EXTRACTION AND CHARTING}

A data extraction form will be developed that charts standard information of both qualitative and quantitative studies included for review (see table 1 ). The chart will be pre-tested by the research team for the first five results. Two researchers will chart the data and any disagreements will be resolved through consensus with a third researcher. The following variables will determine the data to be collected: (1) authors, (2) article title and year of publication, (3) geographical setting in which the study was conducted, (4) study population: interns, residents, registrars, fellows, hospital doctors, specialty trainees, (5) sample size, (6) study design (qualitative, quantitative or mixed method), (7) educational setting (class, ward round, workshop, programme, course, module, lecture, online), (8) educational content (EBM, EBP, evidence-based surgery, EBHC; SDM), (9) delivery (the creation, delivery and analysis of educational interventions) and (10) outcomes (Based on Barr's classification $^{23}$ and may include outcome measures such as: doctors' knowledge, attitudes, practice or patients' experience).

\section{SUMMARY AND REPORTING OF RESULTS}

The study selection procedure will be summarised using a PRISMA flow diagram. ${ }^{22}$ The team will collate, summarise and report all data obtained from the search results to map the number and kinds of EBM courses being run for junior doctors, the number and kinds of SDM training courses for junior doctors and the programmes containing both EBM and SDM training and assessment. Outcomes, where available, will be ascertained by documenting changes in doctors' EBM knowledge, attitudes and practices, and their use of SDM. Patient experiences of the SDM process will also be obtained, where possible. The outcomes, according to Barr's classification ${ }^{23}$ will be evaluated as: Level 1: learners' reaction. Level 2a: modification of attitudes/perceptions. Level 2b: acquisition of knowledge/skills. Level 3: change in behaviour. Level 4a: change in organisational practice. This relates to wider changes in the organisation/delivery of care, attributable to an education programme. Level 4b: benefits to patients/clients. Using the data charting form, a table will be created to describe the study population and design, characteristics of EBM and SDM educational programmes, including programmes that include both; and their outcomes, including patient experience data. Specific EBM and SDM outcomes will be documented within Barr's framework, ${ }^{23}$ such as EBM knowledge scores, EBM attitude surveys, self-reported outcomes, patient-reported SDM outcomes, observer-rated SDM outcomes. Qualitative data (patient and doctor experience) will be reported in narrative form including the use of verbatim, anonymised quotations. The PRISMA-ScR guideline will be used for reporting purposes. ${ }^{20}$ The discussion section will provide an overview of key themes and types of evidence available with reference to the review questions and key groups (patients and junior doctors). We anticipate the study results will provide an invaluable understanding of the gaps that exist in current EBM training programmes, and the degree to which SDM can contribute to such programmes to improve outcomes for patients and doctors. We will refer to the framework developed by Robinson et $a l^{24}$ to identify research gaps in this review by using the elements of our research question (P: Population, C: Concept, Co: Context elements) and the identification of the reason(s) why the gap exists such as (1) insufficient or imprecise information, (2) biased information, (3) inconsistency or unknown consistency and (4) not the right information. 


\section{CONSULTATION}

We plan to use the data and results of the scoping review to support our current research into the outcomes of incorporating SDM into an EBM curriculum for junior doctors in Australia, in the first instance. We are currently interviewing stakeholders in our study (inpatients, junior doctors and consultants in surgical disciplines) to understand more about SDM as part of EBM learning and practice. The results of our scoping review will be shared with these stakeholders as part of the consultation process. Consultations with national and international experts in the fields of EBM and SDM may be sought following our initial analysis of scoping review data. In addition, we will include the views and experiences, as delivered in papers on the topic, of doctors who practice in this field. The insights gained from stakeholders and experts will enhance the results of the scoping review to increase its value to policy makers, practitioners and consumers.

\section{PATIENT AND PUBLIC INVOLVEMENT}

Patients will be invited to comment on involvement in decision-making through individual and group interviews that form part of the wider study from which this scoping review stems. Patient participants will also be invited to comment on the degree to which SDM took place in their care experience and how valuable this was to their care and to the support received by them and their families. These results will be reported alongside details of the other stakeholder consultations, as part of the completed scoping review, adding fine detail to elaborate or clarify any inconsistencies or nuances in review results.

\section{ETHICS AND DISSEMINATION}

The scoping review will synthesise information from publicly available publications and therefore does not require ethical approval. The results from the scoping review will be disseminated through conference presentations and publications in medical journals with all participant data de-identified, and any personal narratives anonymised.

This scoping review will increase our knowledge of how and why EBM and SDM have largely evolved along different pathways, and whether there are opportunities for greater complementarity in the future to inform new training programmes in Australia and beyond. As a result, the scoping review is perceived to have global value and should engender considerable interest within academic and clinical education fields. In this way, we expect the scoping review to present original, innovative evidence of the ways in which the learning and teaching of EBM and SDM can improve patients' care and well-being, clinicians' professional skills, as well as how it might advance the educational paedagogy surrounding EBM and patientcentred care. The broad approach that a scoping review of this nature affords will provide detailed evidence as well as allow us to explore the contributions of different methodologies, such as qualitative research methodologies and mixed methods research, in answering scoping review questions. This should not only contribute to a greater uptake of patient-centred EBM practice where SDM is a key component, but identify key recommendations for enhancing future training of EBM and SDM.

Twitter Yvonne Zurynski @YvonneZurynski and Andrew Davidson @ SydneyBrainSurg

Contributors The authors contributed to the work in accordance with ICMJE recommendations. All authors provided feedback on the concept of the work, and the acquisition, analysis and interpretation of data. MS led the design and conceptualisation of this review and drafted the protocol with primary support from FR, YZ and AD; MS and JC were involved in developing and refining the search strategy and acquisition and management of data; MS and $A D$ were involved in establishing and revising eligibility criteria and data extraction forms. All authors contributed to the final version of the manuscript. All authors agree to be accountable for all aspects of the work.

Funding The authors have not declared a specific grant for this research from any funding agency in the public, commercial or not-for-profit sectors.

Competing interests None declared.

Patient and public involvement Patients and/or the public were not involved in the design, or conduct, or reporting, or dissemination plans of this research.

Patient consent for publication Not required.

Provenance and peer review Not commissioned; externally peer reviewed.

Open access This is an open access article distributed in accordance with the Creative Commons Attribution Non Commercial (CC BY-NC 4.0) license, which permits others to distribute, remix, adapt, build upon this work non-commercially, and license their derivative works on different terms, provided the original work is properly cited, appropriate credit is given, any changes made indicated, and the use is non-commercial. See: http://creativecommons.org/licenses/by-nc/4.0/.

Author note In Australia, a junior doctor refers to: a) Intern: on completing their medical degree, doctors receive provisional registration and enter the workforce as an intern for further training; b) Resident: doctors completing a second or third year of training following internship; c) Registrar: doctors undertaking a medical specialty training programme and d) Fellow: after completion of a specialty training programme, a fellowship provides additional sub-specialty training.Reference: Australian Medical Association. Doctors in Training and Career Advancement (Internet). Australian Capital Territory: AMA Ltd; 2019 https://ama.com.au/careers/ doctors-training-and-career-advancement [Cited 2019 Dec 31].

\section{ORCID iDs}

Mary Simons http://orcid.org/0000-0001-9627-7861

Frances Rapport http://orcid.org/0000-0002-4428-2826

Yvonne Zurynski http://orcid.org/0000-0001-7744-8717

\section{REFERENCES}

1 Sackett DL, Rosenberg WM, Gray JA, et al. Evidence based medicine: what it is and what it isn't. BMJ 1996;312:71-2.

2 Légaré F, Witteman HO. Shared decision making: examining key elements and barriers to adoption into routine clinical practice. Health Aff 2013;32:276-84.

3 Venhuizen G. Can patient centred care plus shared decision making equal lower costs? BMJ 2019;367:15900.

4 Hoffmann TC, Montori VM, Del Mar C. The connection between evidence-based medicine and shared decision making. JAMA 2014;312:1295-6.

5 The CanMEDS frameworkFrank JR, ed. The CanMEDS 2005 physician competency framework. better standards. better physicians. better care. Ottawa: The Royal College of Physicians and Surgeons of Canada, 2005. http://www.ub.edu/medicina unitateducaciomedica/documentos/CanMeds.pdf

6 Albarqouni L, Glasziou P, Hoffmann T. Completeness of the reporting of evidence-based practice educational interventions: a review. Med Educ 2018;52:161-70.

7 Albarqouni L, Hoffmann T, Glasziou P. Evidence-Based practice educational intervention studies: a systematic review of what is taught and how it is measured. BMC Med Educ 2018;18:177. 
8 Hatala R, Guyatt G. Evaluating the teaching of evidence-based medicine. JAMA 2002;288:1110-2.

9 Shaneyfelt T, Baum KD, Bell D, et al. Instruments for evaluating education in evidence-based practice: a systematic review. JAMA 2006;296:1116-27.

10 Simons MR, Zurynski Y, Cullis J, et al. Does evidence-based medicine training improve doctors' knowledge, practice and patient outcomes? A systematic review of the evidence. Med Teach 2019;41:532-8.

11 Thériault G, Bell NR, Grad R, et al. Teaching shared decision making: an essential competency. Can Fam Physician 2019;65:514-6.

12 Stacey D, Légaré $\mathrm{F}$, Lewis $\mathrm{K}$, et al. Decision AIDS for people facing health treatment or screening decisions. Cochrane Database Syst Rev 2017;4:CD001431.

13 Barratt A. Evidence based medicine and shared decision making: the challenge of getting both evidence and preferences into health care. Patient Educ Couns 2008;73:407-12.

14 Rusiecki J, Schell J, Rothenberger S, et al. An innovative shared decision-making curriculum for internal medicine residents: findings from the University of Pittsburgh medical center. Acad Med 2018;93:937-42.

15 Siyam T, Shahid A, Perram M, et al. A scoping review of interventions to promote the adoption of shared decision-making (SDM) among health care professionals in clinical practice. Patient Educ Couns 2019;102:1057-66.
16 Diouf NT, Menear M, Robitaille $\mathrm{H}$, et al. Training health professionals in shared decision making: update of an international environmental scan. Patient Educ Couns 2016;99:1753-8.

17 Akobeng AK. Principles of evidence based medicine. Arch Dis Child 2005;90:837-40.

18 Arksey H, O'Malley L. Scoping studies: towards a methodological framework. Int J Soc Res Methodol 2005;8:19-32.

19 Levac D, Colquhoun H, O'Brien KK. Scoping studies: advancing the methodology. Implement Sci 2010;5:69.

20 Tricco AC, Lillie E, Zarin W, et al. PRISMA extension for scoping reviews (PRISMA-ScR): checklist and explanation. Ann Intern Med 2018;169:467-73.

21 The Joanna Briggs InstituteAromataris E, Munn Z, eds. Joanna Briggs Institute reviewer's manual. The Joanna Briggs Institute, 2017. https://reviewersmanual.joannabriggs.org/

22 Moher D, Liberati A, Tetzlaff J, et al. Preferred reporting items for systematic reviews and meta-analyses: the PRISMA statement. BMJ 2009;339:b2535.

23 Barr H, Freeth D, Hammick M, et al. Evaluations of interprofessional education: a United Kingdom review of health and social care. London: Centre for the Advancement of Interprofessional Education (CAIPE), British Educational Research Association, 2000.

24 Robinson KA, Saldanha IJ, McKoy NA. Development of a framework to identify research gaps from systematic reviews. J Clin Epidemiol 2011;64:1325-30. 
Correction: What are the links between evidence-based

medicine and shared decision-making in training programs

for junior doctors? A scoping review protocol

Simons M, Rapport F, Zurynski Y, et al. What are the links between evidence-based medicine and shared decision-making in training programs for junior doctors? A scoping review protocol. BMJ Open 2020;10:e037225. doi: 10.1136/bmjopen-2020-037225.

The affiliation for Andrew Davidson ${ }^{1}$, Frances Rapport ${ }^{2}$ and Yvonne Zurynski ${ }^{3}$ was incorrect in the published article. The correct affiliations are below.

${ }^{1}$ Department of Clinical Medicine, Macquarie University, North Ryde, NSW 2109, Australia

${ }^{2}$ Professor of Health Implementation Science, Centre for Healthcare Resilience and Implementation Science, Australian Institute of Health Innovation, Macquarie University, North Ryde, NSW 2109, Australia

${ }^{3}$ Associate Professor of Health System Sustainability, Australian Institute of Health Innovation and the NHMRC Partnership Centre for Health System Sustainability, Macquarie University, North Ryde, NSW 2109, Australia

Open access This is an open access article distributed in accordance with the Creative Commons Attribution Non Commercial (CC BY-NC 4.0) license, which permits others to distribute, remix, adapt, build upon this work non-commercially, and license their derivative works on different terms, provided the original work is properly cited, appropriate credit is given, any changes made indicated, and the use is non-commercial. See: http://creativecommons.org/licenses/by-nc/4.0/.

(C) Author(s) (or their employer(s)) 2020. Re-use permitted under CC BY-NC. No commercial re-use. See rights and permissions. Published by BMJ.

BMJ Open 2020;10:e037225corr1. doi:10.1136/bmjopen-2020-037225corr1

D) Check for updates 